\title{
Real-time Investigation of Intracellular Polynucleotide Kinase Using A Cascaded Amplification Circuit
}

Jinhua Shang, Shanshan Yu, Yingying Chen, Yuhui Gao, Chen Hong, Fengzhe Li, Fuan Wang*

College of Chemistry and Molecular Sciences, Wuhan University, Wuhan 430072, P. R.

China

*To whom correspondence should be addressed. E-mail: fuanwang@whu.edu.cn. 


\section{Supporting Information}

\section{Table of Contents}

Table S1. The sequences of oligonucleotides used in the PNK assay

Fig. S1. Schematic illustration of the CDA method S-4

Fig. S2. Schematic illustration of the HCR method

Fig. S3. The kinetic analysis of the CDA-HCR system S-6

Fig. S4. AFM characterization of the CDA-HCR circuit S-7

Fig. S5. Control experiments by removing one hairpin from the CDA-HCR mixture S-8

Fig. S6. The kinetic analysis of the enzyme sensing module

Fig. S7. Optimization of the PNK-targeting CDA-HCR system S-10

Fig. S8. Illustration of the traditional CDA method

Fig. S9. The traditional CDA-mediated PNK assay S-13

Fig. S10. CDA-HCR-mediated PNK analysis in real samples S-14

Fig. S11. Control experiments of the PNK imaging system.

Table S2. Comparison of different amplification strategies for PNK detection S-16

References S-17 
Table S1. The sequences of oligonucleotides used in the PNK assay

\begin{tabular}{cr}
\hline No. & Sequence $\left(\mathbf{5}^{\prime} \rightarrow \mathbf{3}^{\prime}\right)^{*}$ \\
\hline $\mathbf{H}_{\mathbf{1}}$ & 5'-TCT CTA TCA TTA TCT TGC TTC ATC TTC ATC \\
& TCA AGA TAA TGA TAG AGA ACA CTC-3' \\
$\mathbf{H}_{\mathbf{2}}$ & 5'-CTT GCT TCA TCT TCA TCT CCG ACA CTC TCT CTA TCA \\
& TTA TCT TGA GAT GAA GAT GAA GCA AGA TAA TG-3' \\
$\mathbf{H}_{\mathbf{3}}$ & 5'-GAG TGT CGG AGA TGA AGA TGA AGC CAT \\
& CGT GCT TCA TCT TCA TCT CCG-TAMRA-3' \\
$\mathbf{H}_{\mathbf{4}}$ & 5'-GCT TCA TCT TCA TCT CCG GTT TTG \\
& CGG AGA TGA AGA TGA AGC ACG ATG-3' \\
$\mathbf{H}_{\mathbf{5}}$ & 5'-FAM-CAA AAC CGG AGA TGA AGA TGA \\
& AGC TTG CCT GCT TCA TCT TCA TCT CCG-3' \\
$\mathbf{H}_{\mathbf{6}}$ & 5'-GCT TCA TCT TCA TCT CCG ACA CTC \\
& CGG AGA TGA AGA TGA AGC AGG CAA-3' \\
$\mathbf{H}_{\mathbf{1}}$ & 5'-CCC TAC ACG CTA GCT AGA CAA GAT AAG AGT GTT CTC \\
& TAT CAT TAT CTT GTC TAG CTA GCG TGT AGG G-3' \\
T & 5'-GAG TGT TCT CTA TCA TTA TCT T-3' \\
& 5'-GCT TCA TCT TCA TCT CCG ACA CTC-3'
\end{tabular}




\section{Schematic illustration of the CDA method}

As shown in Fig. S1, the catalytic DNA assembly (CDA) system consists of two hairpin structures $\left(\mathrm{H}_{1}\right.$ and $\left.\mathrm{H}_{2}\right)$. In the absence of trigger $\mathbf{T}, \mathrm{H}_{1}$ and $\mathrm{H}_{2}$ can coexist steadily as their cross-reactivity domain was blocked by their intramolecular hybridization to form the hairpin structure. In the presence of $\mathbf{T}$, it would open hairpin $\mathrm{H}_{1}$ and bind to the domain $\mathrm{b}^{*}-\mathrm{a}^{*}-\mathrm{d}^{*}$ of $\mathrm{H}_{1}$ through the toehold-mediated hybridization reaction. The released single-strand domain $c^{*}-b-a$ of the generated intermediate structure $\mathrm{T}-\mathrm{H}_{1}$ then works as a second toehold to stimulate the strand displacement reaction to open hairpin $\mathrm{H}_{2}$. In consequence, a more stable duplex structure $\mathrm{H}_{1}-\mathrm{H}_{2}$ was generated and the trigger sequence $\mathbf{T}$ was released to promote the next round of the CDA reaction. As a result, the introduction of $\mathbf{T}$ fragment can realize the generation of numerous duplex $\mathrm{H}_{1}-\mathrm{H}_{2}$ structures. Meanwhile, the exposed domain d-c* of $\mathrm{H}_{1}-\mathrm{H}_{2}$ can serve as the initiator I of the HCR circuit to stimulate the downstream HCR reaction.

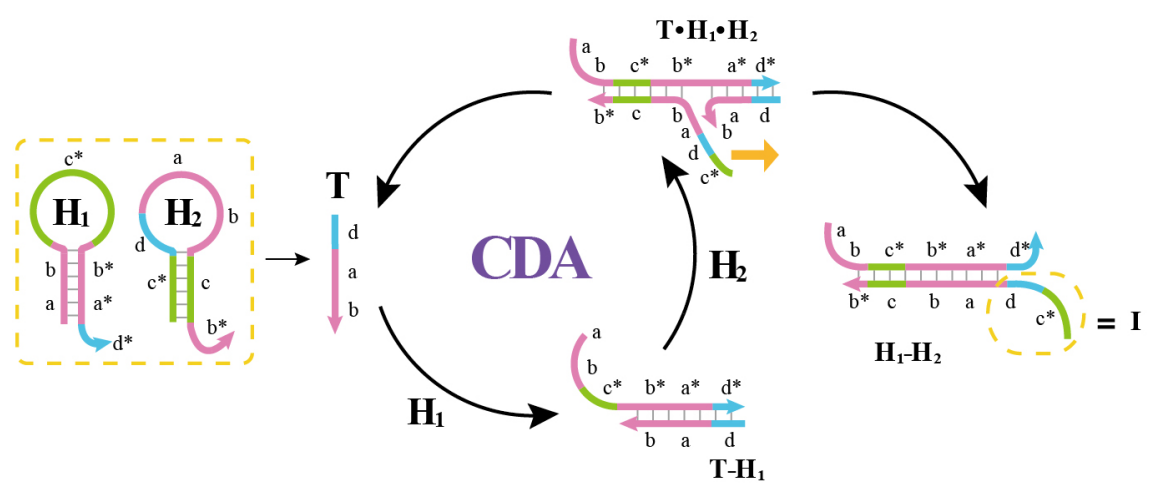

Fig. S1 Schematic representation of the CDA circuit. 


\section{Schematic illustration of the HCR method}

As shown in Fig. S2, we introduce a hybridization chain reaction (HCR) consists of four hairpins $\left(\mathrm{H}_{3}, \mathrm{H}_{4}, \mathrm{H}_{5}\right.$, and $\left.\mathrm{H}_{6}\right)$, which could metastably coexist in the absence of initiator $\mathbf{I}$. $\mathrm{H}_{3}$ contains domains $\mathrm{d}^{*}-\mathrm{c}$ and e-c*, which are complementary to the region d-c* of initiator I and domain $\mathrm{e}^{*}$-c of hairpin $\mathrm{H}_{4}$, respectively. $\mathrm{H}_{4}$ also includes the domain $\mathrm{f}^{*}-\mathrm{c}^{*}$ which is complementary to the region $\mathrm{f}-\mathrm{c}$ of hairpin $\mathrm{H}_{5} . \mathrm{H}_{5}$ consists of domains $\mathrm{f}-\mathrm{c}$ and $\mathrm{g}-\mathrm{c}^{*}$ that are complementary to the region $\mathrm{f}^{*}-\mathrm{c}^{*}$ of $\mathrm{H}_{4}$ and sequence $\mathrm{g}^{*}$-c of hairpin $\mathrm{H}_{6}$, respectively. $\mathrm{H}_{6}$ also includes the analog sequence $\mathrm{d}_{-} \mathrm{c}^{*}$ of the initiator I. Furthermore, the 3' end of $\mathrm{H}_{3}$ and the 5 ' end of $\mathrm{H}_{5}$ are labeled with TAMRA and FAM, respectively. In the presence of $\mathbf{I}$, the domain d-c* opens hairpin $\mathrm{H}_{3}$ through toehold-mediated strand displacement, leading to the formation of intermediate structure $\mathrm{I}-\mathrm{H}_{3}$. The released single strand region e-c* can serve as a new toehold to stimulate the strand displacement reaction with $\mathrm{H}_{4}$, yielding another intermediate structure $\mathrm{I}-\mathrm{H}_{3}-\mathrm{H}_{4}$. Meanwhile, the exposed sequence $\mathrm{f}^{*}-\mathrm{c}^{*}$ of the $\mathrm{I}-\mathrm{H}_{3}-\mathrm{H}_{4}$ is available for hybridization to $\mathrm{H}_{5}$ by binding to the toehold domain $\mathrm{f}$, further opening the hairpin $\mathrm{H}_{5}$ through the strand displacement reaction and forming the $\mathrm{I}-\mathrm{H}_{3}-\mathrm{H}_{4}-\mathrm{H}_{5}$ complex. Analogously, the domain g-c* can then bind to the $\mathrm{H}_{6}$ toehold $\mathrm{g}^{*}$, thus again stimulating the branch migration to open $\mathrm{H}_{6}$ to yield the complex $\mathrm{I}_{-} \mathrm{H}_{3}-\mathrm{H}_{4}-\mathrm{H}_{5}-\mathrm{H}_{6}$. Then the exposed d-c* domain can further react with $\mathrm{H}_{3}, \mathrm{H}_{4}, \mathrm{H}_{5}$, and $\mathrm{H}_{6}$ to generate the growing branched structure $\mathrm{I}-\left(\mathrm{H}_{3}-\mathrm{H}_{4}-\mathrm{H}_{5}-\mathrm{H}_{6}\right)_{\mathrm{N}}$. The generated branched dsDNA nanowires bring fluorophore FAM and TAMRA into proximity and output an amplified FRET signal.

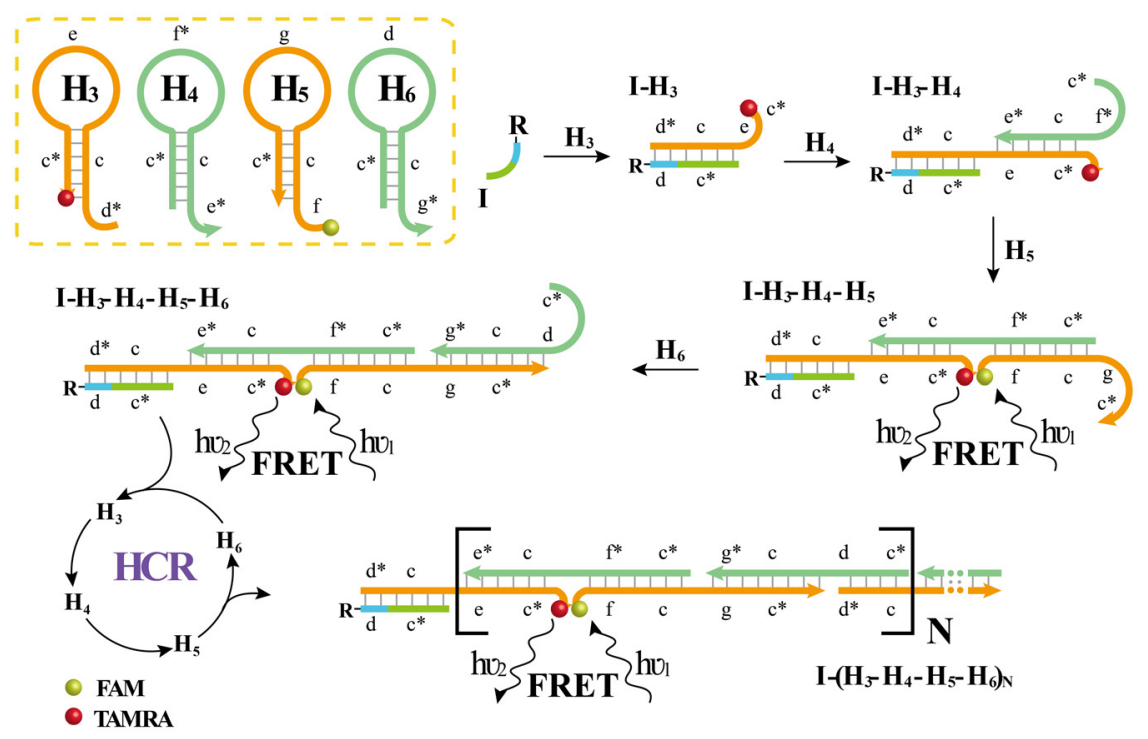

Fig. S2 Schematic representation of the downstream HCR circuit. 


\section{The kinetic analysis of the CDA-HCR system}

For this proof-of-concept study, the as-achieved CDA-HCR amplifier as illustrated in Fig. 1A was first examined by the fluorescence kinetic experiment. As expected, the CDA-HCR mixture shows no fluorescence change without the initiator (curve a, Fig. S3), indicating these DNA probes are metastable without obvious signal leakage (spontaneous reciprocal cross-catalytic reactions). However, when initiator $\mathbf{T}$ was introduced into the CDA-HCR mixture, a dramatical fluorescence change was revealed and it leveled off after $30 \mathrm{~min}$ (curve b, Fig. S3). The result clearly demonstrated the successful coupling of CDA and HCR without signal leakage, and the hierarchical integration of our reciprocal catalytic amplifier with significant signal gain.

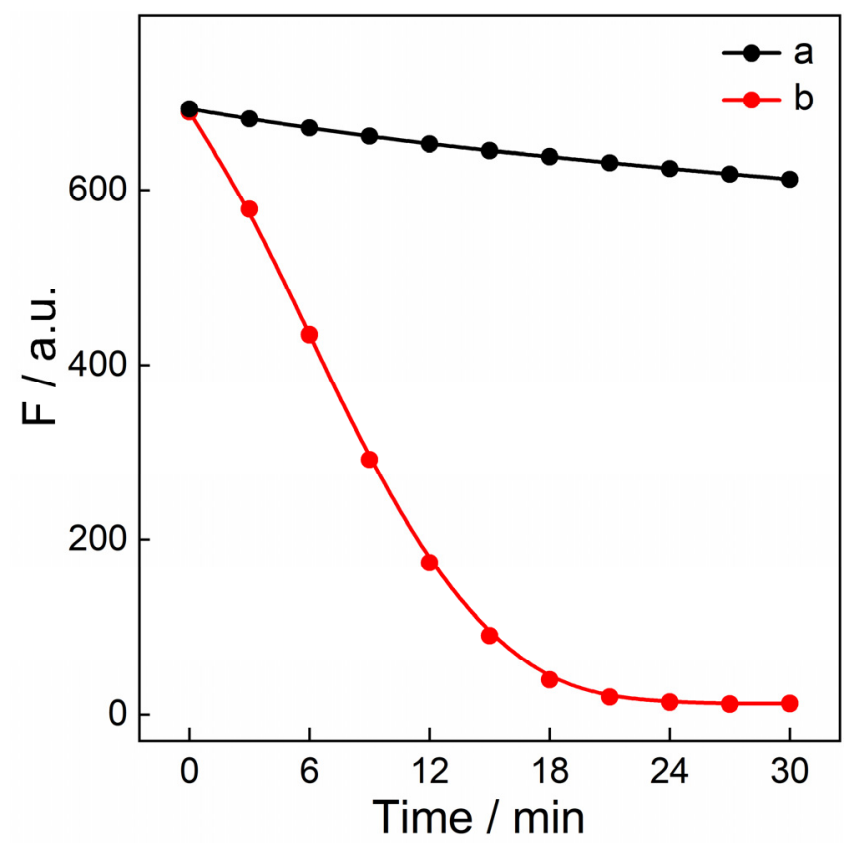

Fig. S3 (A) The fluorescence signal monitoring $\left(\lambda_{\mathrm{ex}}=490 \mathrm{~nm}, \lambda_{\mathrm{em}}=520 \mathrm{~nm}\right)$ of the CDA-HCR circuit without (a) or with (b) $10 \mathrm{nM} \mathbf{T}$. 


\section{AFM characterization of the CDA-HCR circuit}

Atomic force microscopy (AFM) was used to further demonstrate the envisaged characteristics of the CDA-HCR-generated DNA nanostructures. As shown in Fig. S4A, only tiny spots were observed without seeing any assembled nanowire in the absence of trigger $\mathbf{T}$. In contrast, a large number of long DNA nanowires with the height of approximately $1 \mathrm{~nm}$ (Fig. S4C) appeared upon the addition of $\mathbf{T}$ into the system (Fig. S4B). Hence, the AFM images investigated that our designed CDA-HCR amplification method took place as anticipated, which agrees with the results obtained from the fluorescence experiment (Fig. 1B) and non-denaturing polyacrylamide gel electrophoresis (Fig. 1C).
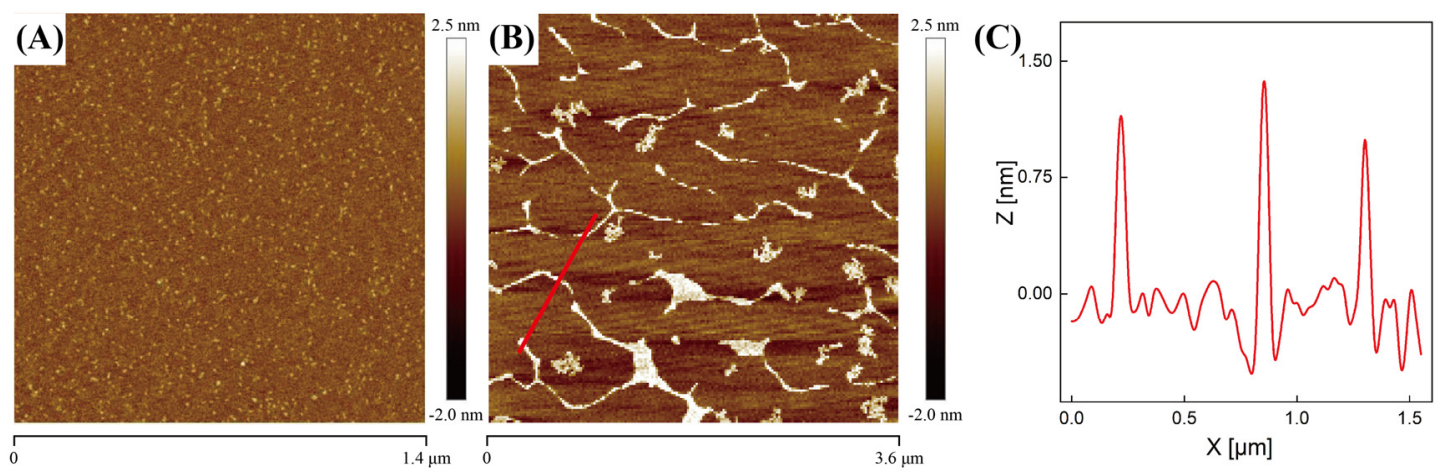

Fig. S4 AFM images of the CDA-HCR system (A) in the absence and (B) in the presence of trigger $\mathbf{T}$. (C) Height profiles of the CDA-HCR-generated dsDNA nanowires as probed by AFM cross-section analysis. The CDA-HCR system was prepared in the reaction buffer that contains $\mathrm{H}_{1}-\mathrm{H}_{6}(100 \mathrm{nM}$ each) and $10 \mathrm{nM}$ trigger T. 


\section{Control experiments by removing one hairpin from the CDA-HCR mixture}

To further investigate the mechanism of the CDA-HCR circuit, one of the non-fluorescent hairpin components $\left(\mathrm{H}_{1}, \mathrm{H}_{2}, \mathrm{H}_{4}\right.$, and $\left.\mathrm{H}_{6}\right)$ was removed from the CDA-HCR system, respectively. As shown in Fig. S5, no obvious fluorescence changes were observed for the trigger-stimulated CDA-HCR circuit by subtracting $\mathrm{H}_{1}$ or $\mathrm{H}_{2}$ from the CDA method (samples a and $\mathrm{b}$, respectively), or by subtracting $\mathrm{H}_{4}$ from the downstream HCR method (sample c). In contrast, a moderate fluorescence response was generated in the trigger-initiated CDA-HCR system without hairpin $\mathrm{H}_{6}$ (sample d). It is reasonable since the CDA circuit was totally impeded without hairpin $\mathrm{H}_{1}$ or $\mathrm{H}_{2}$ and the HCR circuit was similarly blocked without hairpin $\mathrm{H}_{4}$. However, the H6-expelled CDA-HCR circuit can serve as a traditional CDA method that the upstream CDA amplification is intact and the $\mathrm{H}_{6}$-expelled downstream HCR is only used as the signal output function. Hence, both the upstream CDA and downstream HCR reactants are indispensable to the efficient amplification of the CDA-HCR strategy.

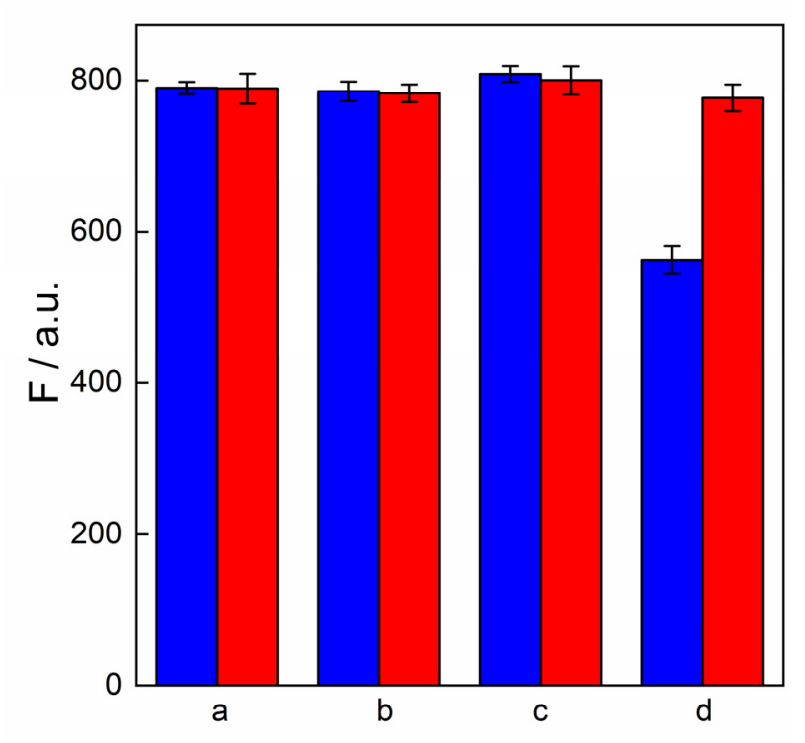

Fig. S5 Fluorescence intensity (at $\lambda=520 \mathrm{~nm}$ ) of the CDA-HCR system upon removing $\mathrm{H}_{1}$ (a), $\mathrm{H}_{2}$ (b), $\mathrm{H}_{4}$ (c), or $\mathrm{H}_{6}$ (d) from the reactants mixture. The blue and red bars represent the fluorescence intensity of the CDA-HCR system with and without $10 \mathrm{nM}$ trigger T, respectively. Error bars were derived from 3 parallel experiments. 


\section{Supporting Information}

\section{The kinetic analysis of the enzyme sensing module}

The kinetic performance of the enzyme sensing module (Fig. S6A) was investigated by the PAGE experiment. First, the kinetic analysis of PNK-involved phosphorylation reaction was carried out by incubating $1 \mathrm{U} / \mathrm{mL}$ polynucleotide kinase (PNK) with hairpin $\mathbf{H}_{\mathbf{T}}$ for varied reaction times, followed by its incubation with 0.05 $\mathrm{U} / \mathrm{mL} \lambda$ exonuclease ( $\lambda$ Exo) under $37^{\circ} \mathrm{C}$ for $60 \mathrm{~min}$ (top, Fig. S6B). In order to analyze the result more rigorously, the gray value of the PAGE image was measured by using Image J and Fiji software (down, Fig. S6B). As shown in Fig. S6B, the band of hairpin $\mathbf{H}_{\mathbf{T}}$ decreased gradually with prolonged phosphorylation time under $37^{\circ} \mathrm{C}$ and disappeared after $90 \mathrm{~min}$. The amount of trigger $\mathbf{T}$ dramatically increased at the beginning of the reaction and then reached a plateau. As shown in Fig. S6C, the kinetic analysis of the $\lambda$ Exo-mediated digestion reaction was also investigated by incubating $1 \mathrm{U} / \mathrm{mL}$ PNK with hairpin $\mathbf{H}_{\mathrm{T}}$ under $37{ }^{\circ} \mathrm{C}$ for $90 \mathrm{~min}$, followed by its incubation with $0.05 \mathrm{U} / \mathrm{mL} \lambda$ Exo for different reaction intervals. The band of $\mathbf{H}_{\mathbf{T}}$ decreased gradually with prolonged cleavage time under $37{ }^{\circ} \mathrm{C}$ and disappeared after $60 \mathrm{~min}$. Meanwhile, the amount of product trigger $\mathbf{T}$ dramatically increased at the beginning of the reaction and then reached a plateau.

(A)
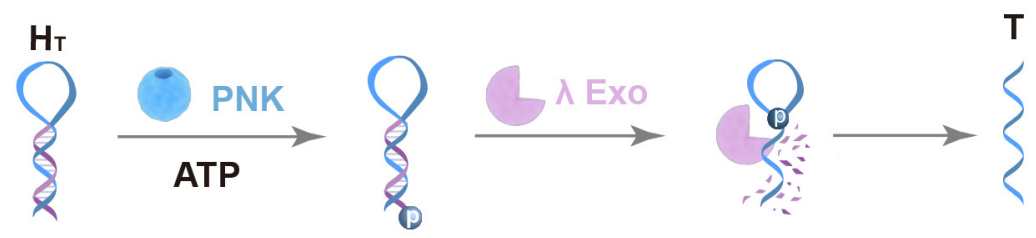

(B)

(C)
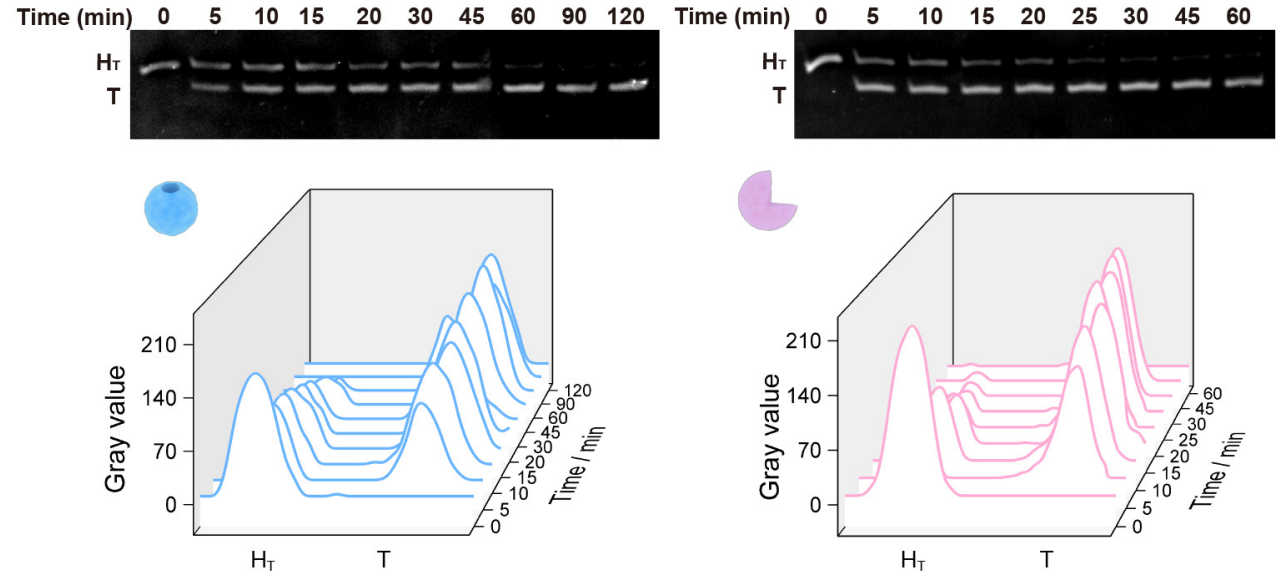

Fig. S6 (A) Schematic working mechanism of the enzyme sensing module. Kinetic analysis of (B) the PNK-involved phosphorylation reaction and (C) the $\lambda$ Exo-mediated cleavage reaction. 


\section{Optimization of the PNK-targeting CDA-HCR system}

The phosphorylation/cleavage condition affects the release efficiency of the trigger $\mathbf{T}$, and determines the performance of PNK. The concentration of ATP was optimized first because it provides phosphate groups during the PNK-mediated phosphorylation reaction. As shown in Fig. S7A, the fluorescence response gradually increases with the increase of ATP concentration, and reaches the maximum at $1 \mu \mathrm{M}$. However, as the ATP concentration increases further, the fluorescence signal response decreases, which may be caused by the steric effect. Hence, $1 \mu \mathrm{M}$ was chosen as the optimized ATP concentration. Then, the concentration of $\lambda$ exonuclease ( $\lambda$ Exo) was optimized to ensure the high efficiency of the digestion reaction. As shown in Fig. S7B, the fluorescence response gradually increases with the increasing concentration of $\lambda$ Exo and reaches a plateau after $0.05 \mathrm{U} / \mathrm{mL}$. Thus, $0.05 \mathrm{U} / \mathrm{mL}$ was chosen as the optimized $\lambda$ Exo concentration. Afterward, the optimized phosphorylation time was investigated by incubating a fixed concentration of PNK with hairpin $\mathbf{H}_{\mathbf{T}}$ for different reaction time intervals. As shown in Fig. S7C, the fluorescence response gradually increases with the prolonged incubation time and reaches the plateau at $90 \mathrm{~min}$. Hence, $90 \mathrm{~min}$ was chosen as the optimized phosphorylation reaction time. At last, the PNK incubation temperature was optimized at a series of reaction temperatures $(25,35,37$, and $40{ }^{\circ} \mathrm{C}$ ). As shown in Fig. S7D, the assay executed at $37^{\circ} \mathrm{C}$ exhibits the highest fluorescence response, which was chosen as the optimized incubation temperature. In summary, the optimized concentration of ATP and $\lambda$ Exo were $1 \mu \mathrm{M}$ and $0.05 \mathrm{U} / \mathrm{mL}$, respectively. The optimized incubation time was chosen as $90 \mathrm{~min}$ and the optimized temperature was chosen as $37^{\circ} \mathrm{C}$. 

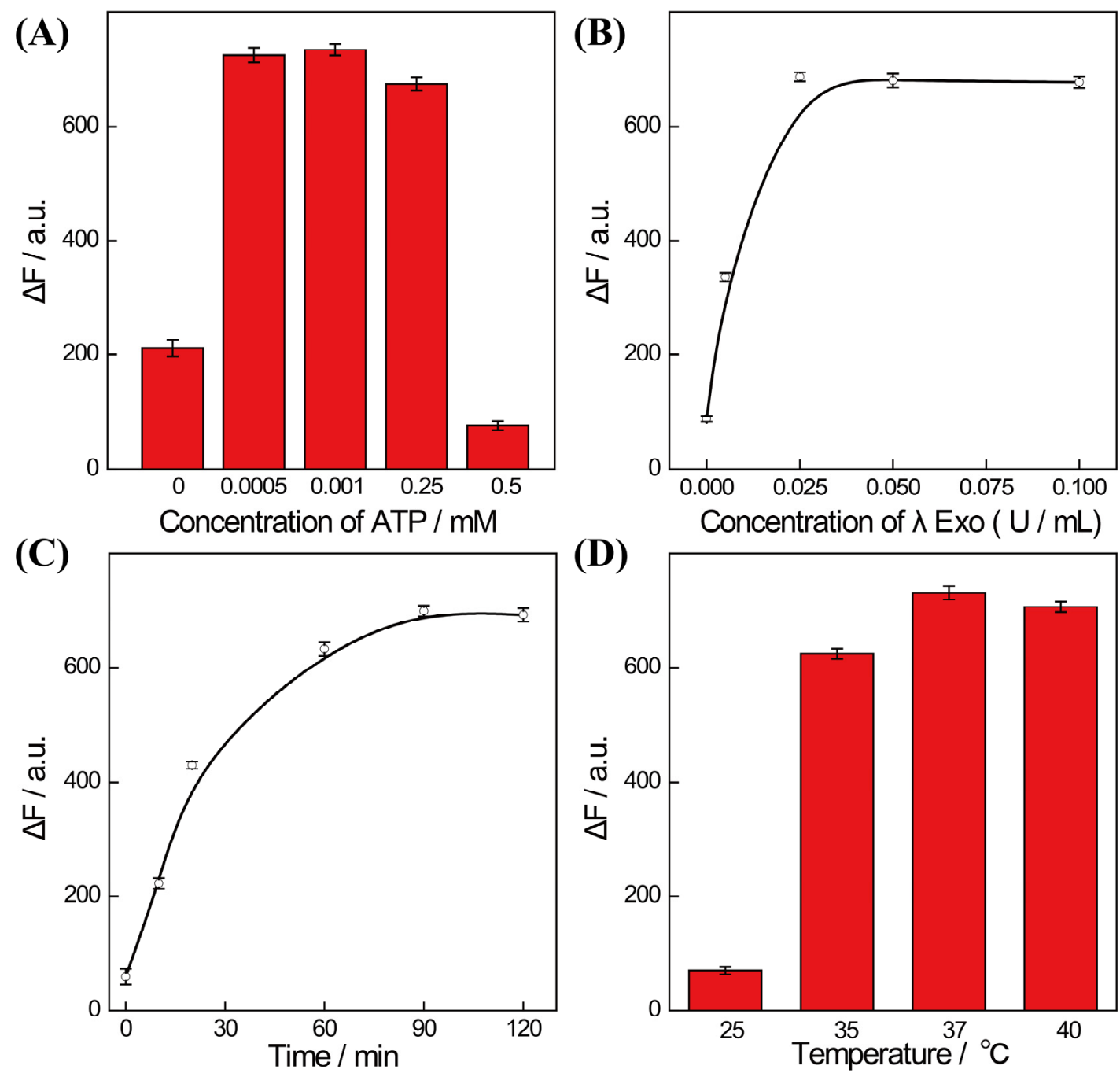

Fig. S7 Optimization of (A) the ATP concentration, (B) the $\lambda$ Exo concentration, (C) phosphorylation incubation time, (D) phosphorylation incubation temperature. Error bars were derived from 3 parallel experiments. 


\section{Illustration of the traditional CDA method}

As shown in Fig. S8A, the H6-expelled CDA-HCR circuit represents the traditional CDA method. Here $\mathbf{T}$ triggers the integral signal amplification of the upstream CDA circuit while the $\mathrm{H}_{6}$-expelled HCR only executes the signal readout function, giving rise to the FAM/TAMRA hybrid with a ratio corresponding to $1: 1$. In the absence of trigger $\mathbf{T}$, the fluorescence intensity of the CDA mixture nearly unchanged (curve a, Fig. S8B), indicating that these hairpin reactants could metastably coexist without undesired cross-hybridization reaction or signal leakage. A moderate fluorescence response was observed for the trigger-initiated CDA strategy (curve b, Fig. S8B), which was reasonable since the amplification of the traditional CDA $(1 / \mathrm{N})$ is significantly lower than that of the CDA-HCR method $\left(1 / \mathrm{N}^{2}\right)$.

(A)

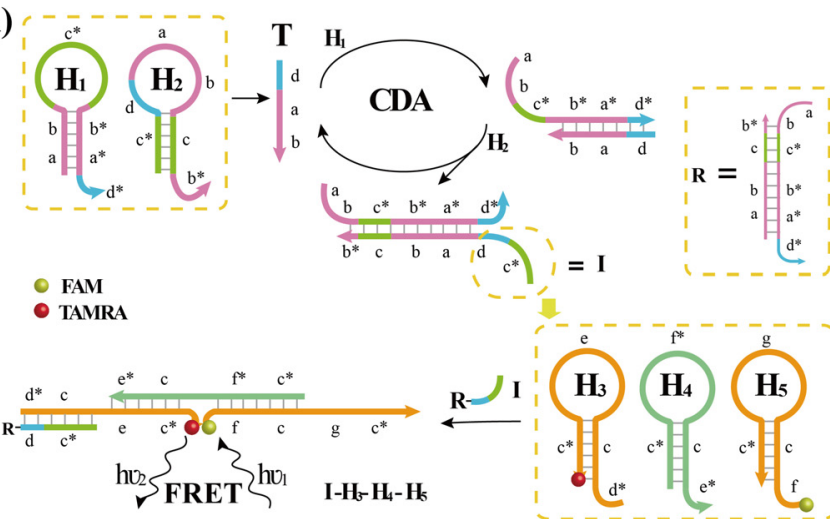

(B)

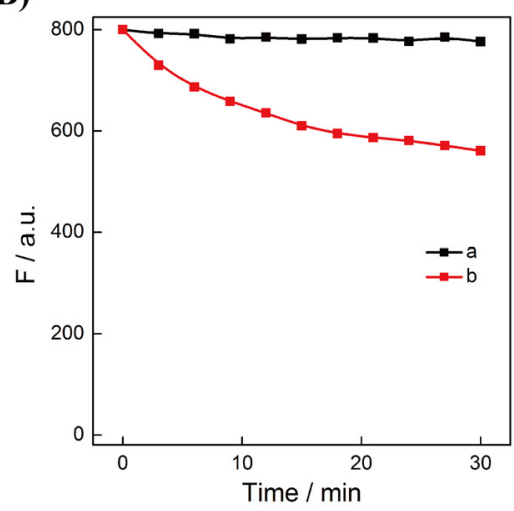

Fig. S8 (A) The scheme of the traditional CDA circuit. (B) Time-dependent fluorescence intensity of the CDA-HCR circuit (at $\lambda=520 \mathrm{~nm}$ ) without (a) and with (b) $10 \mathrm{nM}$ trigger $\mathbf{T}$. 


\section{The traditional CDA-mediated PNK assay}

The traditional CDA method was further applied for analyzing the activity of PNK under the optimized experiment condition. Fig. S9A shows the fluorescence spectra of the CDA amplifier incubating with different concentrations of PNK. The fluorescence intensity of FAM gradually decreased with the increasing concentrations of PNK. As shown in Fig. S9B, a good linear relationship was observed for PNK ranging from $0.0125-1.25 \mathrm{U} / \mathrm{mL}$ with a derived equation expressed as $\Delta \mathrm{F}=179.69 \mathrm{c}$ $+3.44\left(\mathrm{R}^{2}=0.994\right)$.
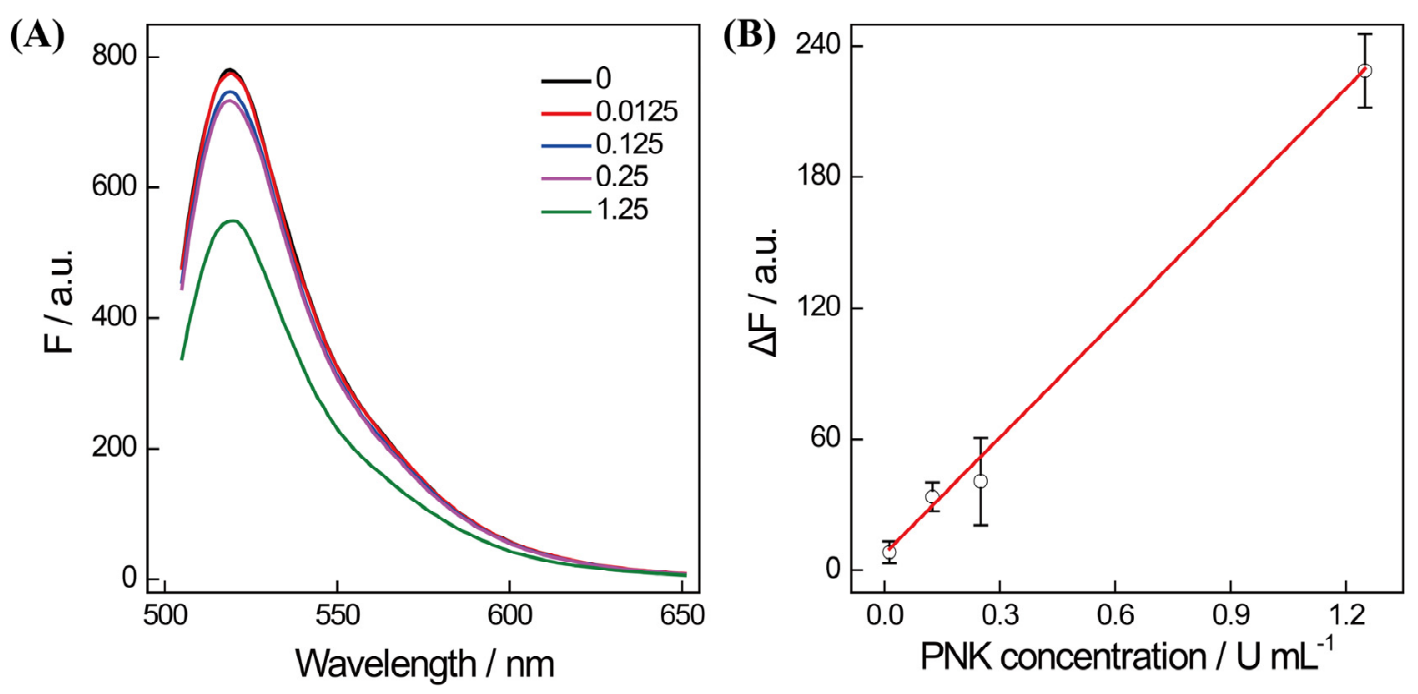

Fig. S9 (A) Fluorescence spectra of the traditional CDA-mediated PNK biosensor with different concentrations of PNK enzyme. (B) The linear calibration curve of the traditional CDA-mediated PNK analysis. 


\section{CDA-HCR-mediated PNK analysis in real samples}

The proposed CDA-HCR-based PNK assay was further carried out to analyze the activity of endogenous PNK from HeLa cells lysate. The total amount of protein in HeLa cells lysate was measured by using the BCA protein assay according to the manufacturer's manual. After adding a certain amount of HeLa cells lysate to the $\mathbf{H}_{\mathbf{T}}$ incubation reaction, hairpin $\mathbf{H}_{\mathbf{T}}$ was mixed with $\lambda$ Exo for digestion. Then the activity of endogenous PNK was analyzed by using the CDA-HCR method. As shown in Fig. S10, the fluorescence variation intensified with the increased content of extracted total proteins in HeLa cells lysate. There revealed a linear relationship between fluorescence variation and the total protein amount in the range of 75-190 $\mathrm{ng}$. According to the $3 \sigma / \lambda$ method, the limit of detection was calculated to be $6.5 \mathrm{ng}$ for the CDA-HCR-mediated total protein assay. The result obviously demonstrates that our designed CDA-HCR method is suitable for analyzing PNK activity in real complex biological samples, thus holding excellent promise for clinical diagnosis.

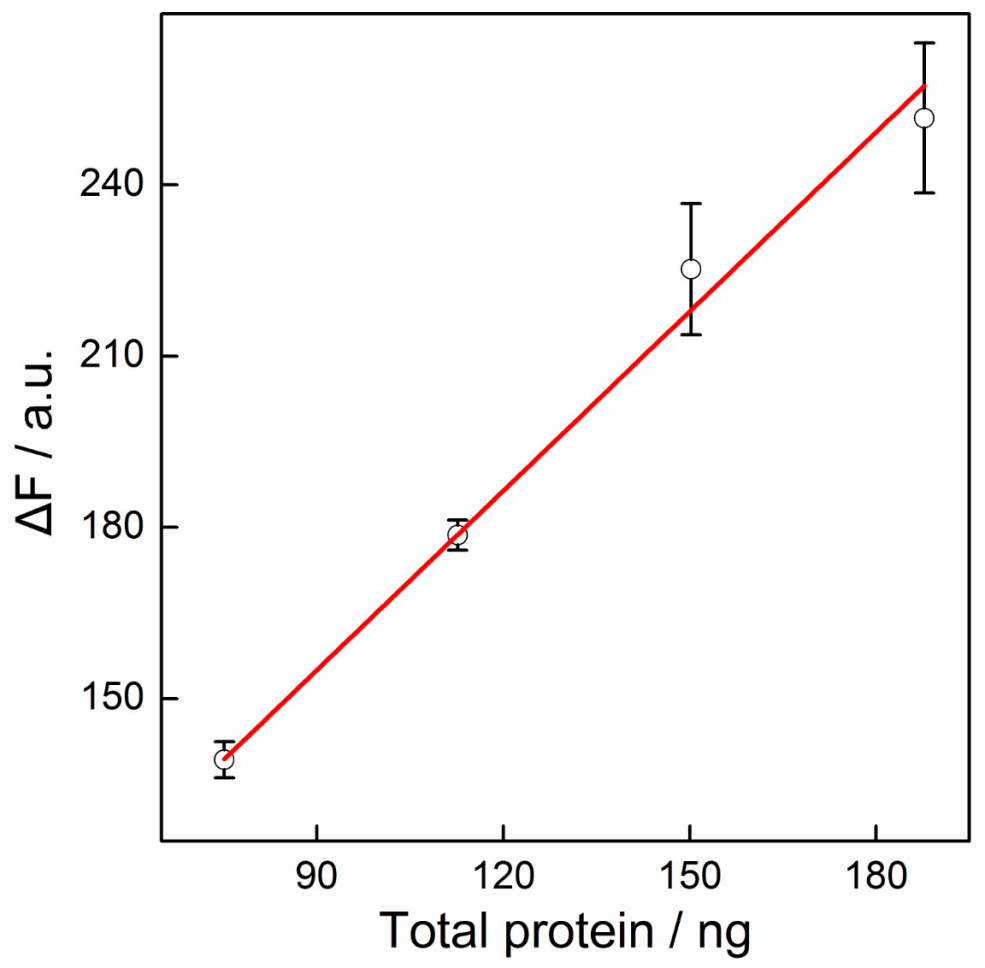

Fig. S10 The linear plotting relationship between the fluorescence changes $\left(\Delta \mathrm{F}=\mathrm{F}_{0}-\right.$ F) and total protein contents. 


\section{Supporting Information}

\section{Control experiments of the PNK imaging system}

To further demonstrate the amplified mechanism of the CDA-HCR-mediated intracellular PNK imaging system, several control experiments were carried out (Fig. S11). As compared with the $F_{A} / F_{D}$ value of the integral CDA-HCR system (sample a, Fig. S11), an obviously weaker $F_{A} / F_{D}$ signal was observed in HeLa cells by removing $\mathbf{H}_{\mathbf{T}}$ (sample b, Fig. S11), or $\lambda$ Exo (sample c, Fig. S11), respectively. The result indicated that the trigger sequence $\mathbf{T}$ cannot be successfully released in the absence of $\lambda$ Exo or $\mathbf{H}_{\text {T. }}$. Also, it revealed that only the intact CDA-HCR-mediated PNK biosensor can provide efficient amplification for in situ imaging of intracellular PNK in living HeLa cells.

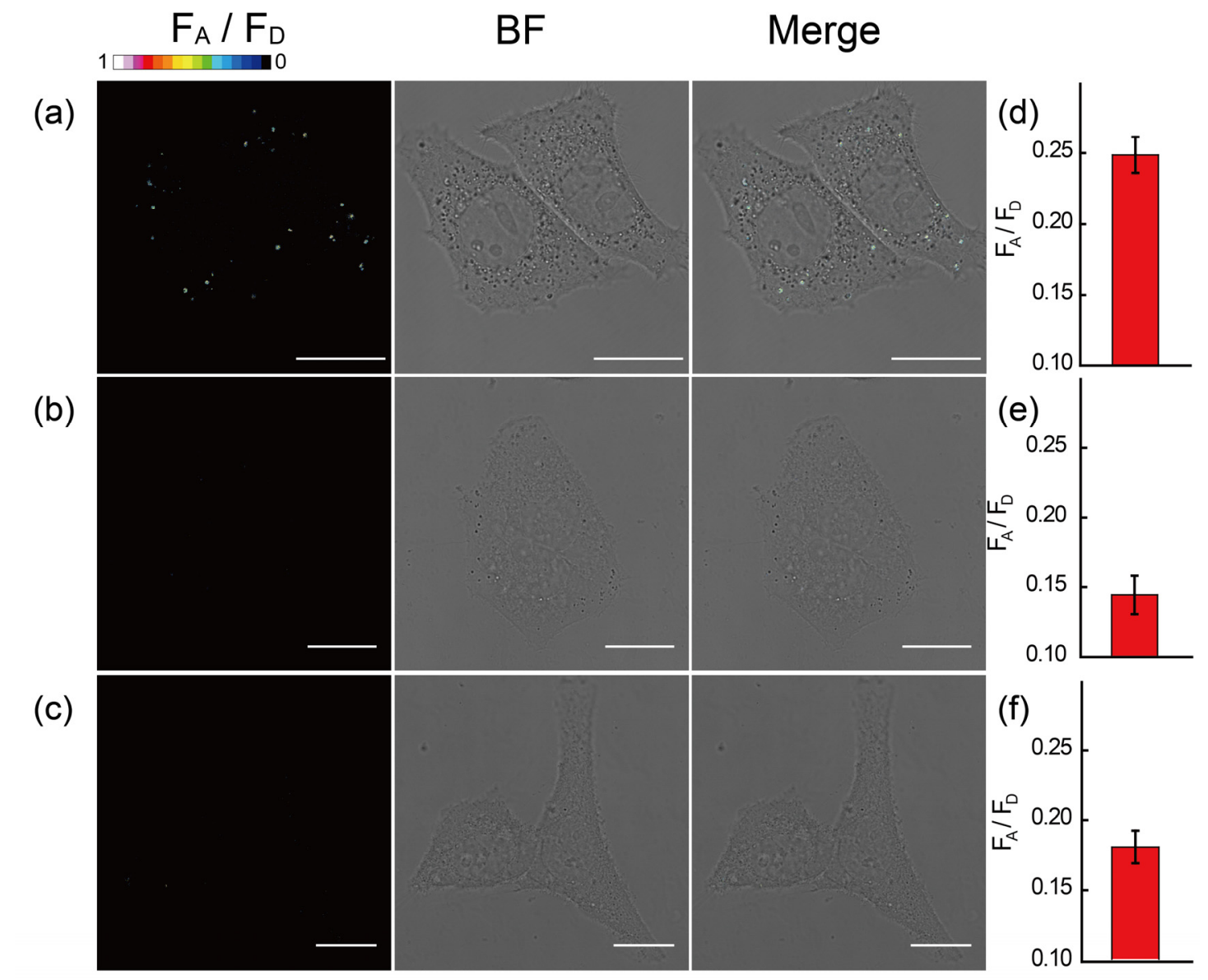

Fig. S11 Confocal laser scanning microscopy imaging of intracellular PNK in HeLa cells by (a) the intact CDA-HCR method, (b) $\mathbf{H}_{\mathrm{T}}$-removed CDA-HCR method, (c) $\lambda$ Exo-removed CDA-HCR method, (d) Statistical histogram analysis of (a). (e) Statistical histogram analysis of (b). (f) Statistical histogram analysis of (c). The scale bar is $20 \mu \mathrm{m}$. 
Table S2 Comparison of different amplification strategies for PNK detection

\begin{tabular}{|c|c|c|c|}
\hline Methods & $\begin{array}{l}\text { Detection range } \\
(\mathrm{U} / \mathrm{mL})\end{array}$ & $\begin{array}{l}\text { Detection limit } \\
(\mathrm{U} / \mathrm{mL})\end{array}$ & Ref. \\
\hline $\begin{array}{l}\text { Fluorescence assay based on the allosteric aptamer probe } \\
\qquad \text { (AAP) }\end{array}$ & $0-1$ & 0.01 & (1) \\
\hline $\begin{array}{l}\text { Electrochemical detection based on target-assisted ligation } \\
\text { reaction coupled with silver nanoparticles }\end{array}$ & $0.1-100$ & 0.01 & (2) \\
\hline $\begin{array}{l}\text { Colorimetric assay based on the G-quadruplex/hemin } \\
\text { DNAzyme }\end{array}$ & $0-15$ & 0.01 & (3) \\
\hline $\begin{array}{l}\text { Chemiluminescence assay based on the rolling circle } \\
\text { amplification }\end{array}$ & $0.0001-100$ & $2.20 \times 10^{-4}$ & (4) \\
\hline $\begin{array}{l}\text { Fluorescence sensing based on DNA/polydopamine } \\
\text { nanospheres platform }\end{array}$ & $0-10$ & 0.01 & $(5)$ \\
\hline $\begin{array}{l}\text { Fluorescence assay based on terminal deoxynucleotidyl } \\
\text { transferase-activated nicking enzyme amplification reaction }\end{array}$ & $0.001-0.1$ & $4 \times 10^{-4}$ & $(6)$ \\
\hline $\begin{array}{l}\text { Photoelectrochemical biosensor based on the } \mathrm{TiO}_{2} / \mathrm{g}^{-} \\
\qquad \mathrm{C}_{3} \mathrm{~N}_{4} / \mathrm{CdS} \text { Nanocomposite }\end{array}$ & $0-1$ & $6.9 \times 10^{-5}$ & (7) \\
\hline Fluorescence assay based on conventional CDA & $0.0125-1.25$ & 0.0125 & $\begin{array}{l}\text { This } \\
\text { work }\end{array}$ \\
\hline Fluorescence assay based on CDA-HCR amplifier & $2.5 \times 10^{-4}-2.5 \times 10^{-1}$ & $2.5 \times 10^{-4}$ & $\begin{array}{l}\text { This } \\
\text { work }\end{array}$ \\
\hline
\end{tabular}




\section{Supporting Information}

\section{References}

(1) Gao, M.; Guo, J.; Song, Y.; Zhu, Z.; Yang, C. Detection of T4 Polynucleotide Kinase via Allosteric Aptamer Probe Platform. ACS Appl. Mater. Interfaces 2017, 9, 38356-38363.

(2) Jiang, Y.; Cui, J.; Zhang, T.; Wang, M.; Zhu, G.; Miao, P. Electrochemical Detection of T4 Polynucleotide Kinase based on Target-Assisted Ligation Reaction Coupled with Silver Nanoparticles. Anal. Chim. Acta 2019, 1085, 85-90.

(3) Liu, H.; Ma, C.; Wang, J.; Chen, H.; Wang, K. Label-Free Colorimetric Assay for T4 Polynucleotide Kinase/Phosphatase Activity and its Inhibitors based on G-quadruplex/Hemin DNAzyme. Anal. Biochem. 2017, 517, 18-21.

(4) Tang, W.; Zhu, G.; Zhang, C. Sensitive Detection of Polynucleotide Kinase using Rolling Circle Amplification-Induced Chemiluminescence. Chem. Commun. 2014, 50, 4733-4735.

(5) Cen, Y.; Deng, W.; Yu, R.; Chu, X. Sensitive Fluorescence Sensing of T4 Polynucleotide Kinase Activity and Inhibition based on DNA/Polydopamine Nanospheres Platform. Talanta 2018, 180, 271-276.

(6) Du, Y.; Wang, S.; Li, X.; Wang, Y.; Tang, A.; Kong, D. Terminal Deoxynucleotidyl Transferase-Activated Nicking Enzyme Amplification Reaction for Specific and Sensitive Detection of DNA Methyltransferase and Polynucleotide kinase. Biosens. Bioelectron. 2019, 145, 111700.

(7) Li, P.; Cao, Y.; Mao, C.; Jin, B.; Zhu, J. A TiO2/g-C3N4/CdS Nanocomposite-Based Photoelectrochemical Biosensor for Ultrasensitive Evaluation of T4 Polynucleotide Kinase Activity. Anal. Chem. 2019, 91, 1563-1570 